\title{
Analyst
}

CORRECTION

(a)

CrossMark

Cite this: Analyst, 2015, 140, 6758

DOI: 10.1039/c5an90071a

www.rsc.org/analyst
View Article Online

View Journal | View Issue

\section{Correction: Towards high-throughput microfluidic Raman-activated cell sorting}

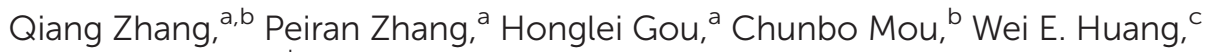
Menglong Yang, ${ }^{* d}$ Jian $\mathrm{Xu}^{*^{\mathrm{a}}}$ and $\mathrm{Bo} \mathrm{Ma}^{*^{\mathrm{a}}}$

Correction for 'Towards high-throughput microfluidic Raman-activated cell sorting' by Qiang Zhang et al., Analyst, 2015, DOI: 10.1039/c5an01074h.

The original article contained an error in the affiliation of author Wei E. Huang to the addresses associated with this work. This author should be attributed to address c only. The correct list of authors and affiliations is above.

The Royal Society of Chemistry apologises for these errors and any consequent inconvenience to authors and readers.

${ }^{a}$ Single-Cell Center, CAS Key Laboratory of Biofuels and Shandong Key Laboratory of Energy Genetics, Qingdao Institute of Bioenergy and Bioprocess Technology, Chinese Academy of Sciences, Qingdao, China. E-mail: xujian@qibebt.ac.cn, mabo@qibebt.ac.cn

${ }^{b}$ College of Chemical Science and Engineering, Qingdao University, Qingdao, China

${ }^{c}$ Department of Engineering Science, University of Oxford, Parks Road, Oxford OX1 3PJ, UK

${ }^{d}$ Public Laboratory and CAS Key Laboratory of Biofuels, Qingdao Institute of Bioenergy and Bioprocess Technology, Chinese Academy of Sciences, Qingdao, China. E-mail:yangml@qibebt.ac.cn 